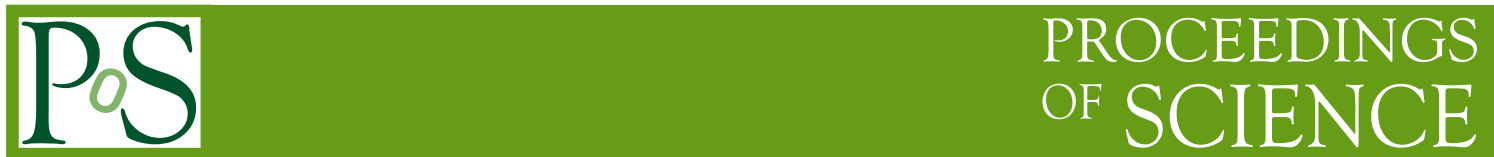

\title{
$B_{s}$ Decays and Mixing
}

\section{Jonathan L. Rosner}

Enrico Fermi Institute and Department of Physics

University of Chicago

E-mail: rosner@hep.uchicago.edu

\section{Michael Gronau}

Department of Physics, Technion - Israel Institute of Technology

Haifa, Israel

E-mail: gronauephysics.technion.ac.il

Theoretical remarks are offered regarding recent hadron collider results on the mixing and decays of $B_{s}$ mesons. Topics covered include: (1) CP-violating mixing in $B_{s}\left(\bar{B}_{s}\right) \rightarrow J / \psi \phi$, (2) the D0 dimuon charge asymmetry, (3) information from triple products, (4) $B_{s} \rightarrow J / \psi f_{0}$, (5) new physics constraints, (6) some illustrative new physics scenarios.

The 13th International Conference on B-Physics at Hadron Machines - Beauty2011, April 04-08, 2011

Amsterdam, The Netherlands

*Speaker. 


\section{Introduction}

Recent results on $B_{s}$ decays and mixing have been presented by the CDF and D0 Collaborations at the Fermilab Tevatron and the LHCb Collaboration at CERN. We begin by discussing CP-violating mixing in $B_{S}\left(\bar{B}_{s}\right) \rightarrow J / \psi \phi$. Experiments at CDF and D0 suggested a mixing phase $\beta_{s}$ much larger than that in the Standard Model (SM). With such a large phase, we pointed out that time-dependent decays should display explicit time-dependence [1]. We update that analysis in Section 2.

The D0 Collaboration has presented evidence for a charge asymmetry in same-sign dimuons produced in $\bar{p} p$ collisions at $\sqrt{s}=1.96 \mathrm{TeV}$ [2]. We suggest in Section 3 a test of whether this asymmetry is due to decays of $b$ quarks, as claimed, or background sources such as kaons [3].

In Section 4 we discuss what triple products in $B_{(s)} \rightarrow V_{1} V_{2}$ actually measure. The answer [4] is CP violation, but only under certain conditions. The study of $B_{s} \rightarrow J / \psi f_{0}$, mentioned in Section

5 , avoids the angular analysis needed to interpret $B_{s} \rightarrow J / \psi \phi$. In Section 6, we note constraints on new physics, and comment in Section 7 on a couple of scenarios for consideration should any hints for physics beyond the SM be borne out by further tests. We conclude in Section 8.

\section{CP violation in interference between $B_{s}-\bar{B}_{s}$ mixing and $B_{s} \rightarrow J / \psi \phi$ decay}

For formalism we refer to [5]. $B_{s}-\bar{B}_{s}$ mixing is expected to be dominated by the top quark in box graphs. The observed values $\Delta m_{s}=(17.77 \pm 0.10 \pm 0.07) \mathrm{ps}^{-1}$ (CDF [6]) and (17.63 $\pm 0.11 \pm$ $0.04) \mathrm{ps}^{-1}$ (LHCb [7]) agree with SM predictions. Denoting

$$
\left|B_{s L}\right\rangle=p\left|B_{s}\right\rangle+q\left|\bar{B}_{s}\right\rangle ;\left|B_{s H}\right\rangle=p\left|B_{s}\right\rangle-q\left|\bar{B}_{s}\right\rangle,
$$

we expect for $\Delta \Gamma \ll \Delta m, q / p \simeq \exp \left(2 i \beta_{s}\right), \beta_{s}^{\mathrm{SM}}=-\operatorname{Arg}\left(-V_{t s}^{*} V_{t b} / V_{c s}^{*} V_{c b}\right)=(1.04 \pm 0.05)^{\circ}$ [5]. The $\mathrm{SM} B_{s} \rightarrow J / \psi \phi \mathrm{CP}$ asymmetry then should be governed by the small mixing phase $\phi_{M}=-2 \beta_{s}^{\mathrm{SM}}$.

In 2008, CDF [8] and D0 [9] favored a mixing phase differing from $-2 \beta_{s}^{\mathrm{SM}}$ by $\sim 2.2 \sigma$ based on the decay $B_{s} \rightarrow J / \psi \phi$. At that time we pointed out that such a large mixing phase (the illustrative value was then $\phi_{M}=-44^{\circ}$ [8]) would imply detectable time-dependence of angular distribution coefficients, differing for tagged $B_{s}$ and $\bar{B}_{s}[1]$.

We review the discussion briefly. For a CP test, one tags the flavor at $t=0$, denoting $\eta= \pm 1$ for a tagged $\left(B_{s}, \bar{B}_{s}\right)$. The coefficients of helicity amplitudes $\left|A_{\|}\right|^{2},\left|A_{\perp}\right|^{2}$ describing different angular dependences are denoted by $\mathscr{T}_{+}, \mathscr{T}_{-}$, where

$$
\mathscr{T}_{ \pm} \equiv e^{-\Gamma t}\left[\cosh (\Delta \Gamma t) / 2 \mp \cos \left(\phi_{M}\right) \sinh (\Delta \Gamma t) / 2 \pm \eta \sin \left(\phi_{M}\right) \sin \left(\Delta m_{s} t\right)\right] .
$$

Taking $\phi_{M}=-44^{\circ}, \Delta \Gamma / \Gamma=0.228$, and assuming the tagging $\eta$ to be diluted by a factor of 0.11 , we concluded that wiggles should be distinguishable between the $B_{s}$-tagged and $\bar{B}_{s}$-tagged $\mathscr{T}_{ \pm}$ distributions. We advocated making such a plot as evidence for CP violation in $B_{s} \rightarrow J / \psi \phi$ at a level beyond the SM. Here we update our estimate of $t$-dependence, finding the oscillations a bit smaller, but still visible. We take $\phi_{M}=(-39 \pm 17)^{\circ}$ based on an average between CDF [10,11] and D0 [12] values, choose $\Delta \Gamma / \Gamma=0.143$ based on an average between CDF $(0.075 \pm 0.035 \pm 0.010)$ and D0 $(0.15 \pm 0.06 \pm 0.01)$, and continue to assume a dilution factor of $11 \%$. The resulting plot is shown in Fig. 1. 


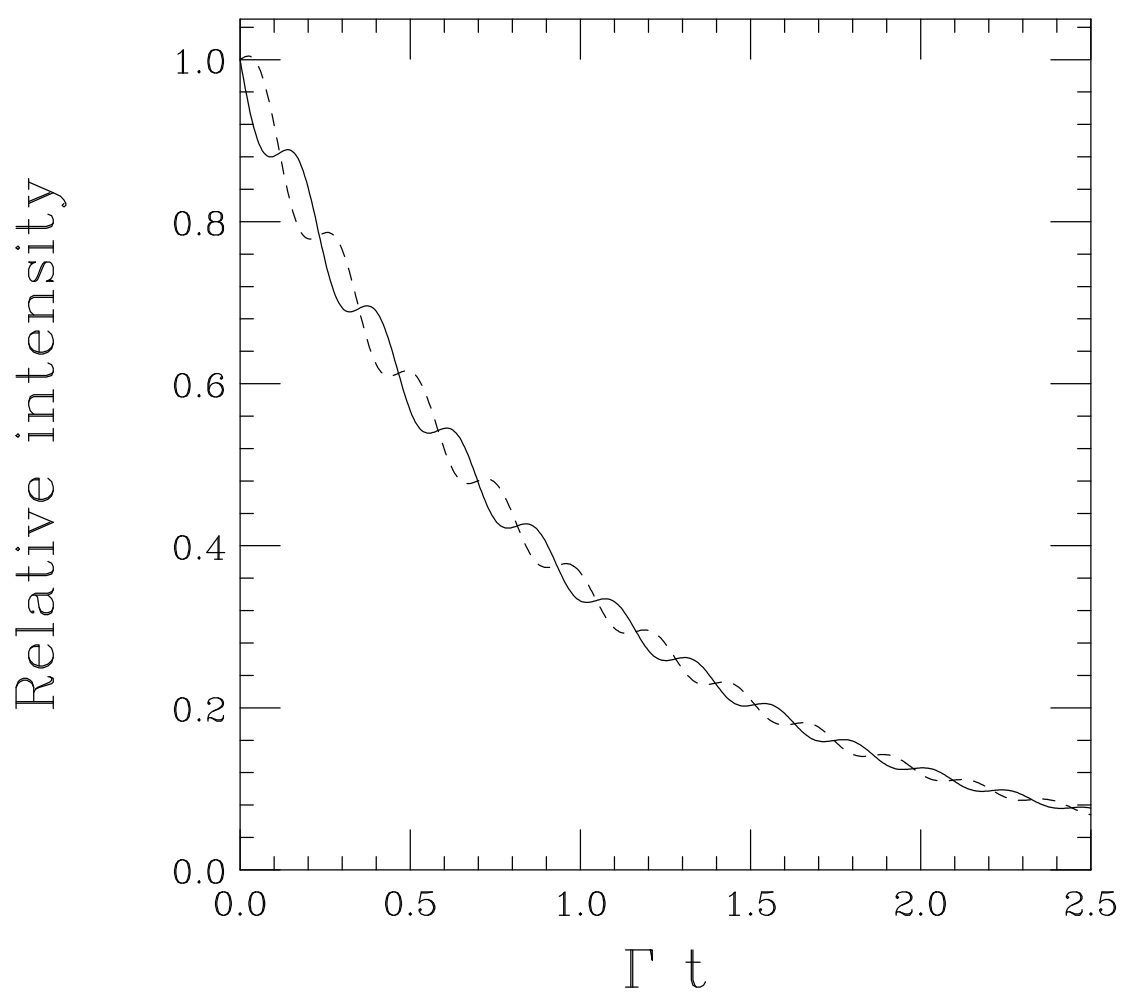

Figure 1: Relative intensities of $\mathscr{T}_{+}$signals as functions of $\Gamma t$, for $B_{s}$ tags (solid) and $\bar{B}_{s}$ tags (dashed). This figure represents an update of a similar one in Ref. [1].

At this Conference, $\mathrm{LHCb}$ presented data restricting $\phi_{M}$ to the range $[-2.7,-0.5][13](68 \%$ c.1.), $1.2 \sigma$ from the SM. We are eagerly awaiting data from ATLAS and CMS.

\section{D0 dimuon asymmetry - Is it due to $b$ 's? $K$ 's?}

The SM predicts a small asymmetry in the yield of same-sign muon pairs due to $b \bar{b}$ production followed by meson $\Leftrightarrow$ antimeson oscillation: $A_{s l}^{b} \equiv \frac{N^{++}-N^{--}}{N^{++}+N^{--}}=(-2.0 \pm 0.3) \times 10^{-4}$ [14]. The D0 Collaboration reports a much larger value, $A_{s l}^{b}=(-9.57 \pm 2.51 \pm 1.46) \times 10^{-3}$, nearly 50 times the $\mathrm{SM}$ value [2]. (CDF is not ready to report such a measurement but has quoted a new average mixing parameter $\bar{\chi}[15]$.)

D0 has interpreted its result as $3.2 \sigma$ evidence for $\mathrm{CP}$ violation in neutral $B$ mixing. They have performed 16 systematic checks for which their results are found consistent with their nominal ones. Estimating the correct kaon decay backgrounds is crucial.

We have suggested a test [3] to see if a smaller asymmetry is obtained in a sample depleted in $b \bar{b}$ pairs. If one reduces the maximum allowed impact parameter of muon tracks, the signal should vanish more rapidly than background. The effect of our suggestion, an impact parameter cut of $b<100 \mu \mathrm{m}$, is not yet known to us.

We denote quantities in the $B$ rest frame with an asterisk $\left(^{*}\right)$ and those in the lab frame with none. The lab energy of the $B$ is $E_{B}=\gamma m_{B}=m_{B} / \sqrt{1-\beta^{2}}$. Muon angles with respect to the $B$ 


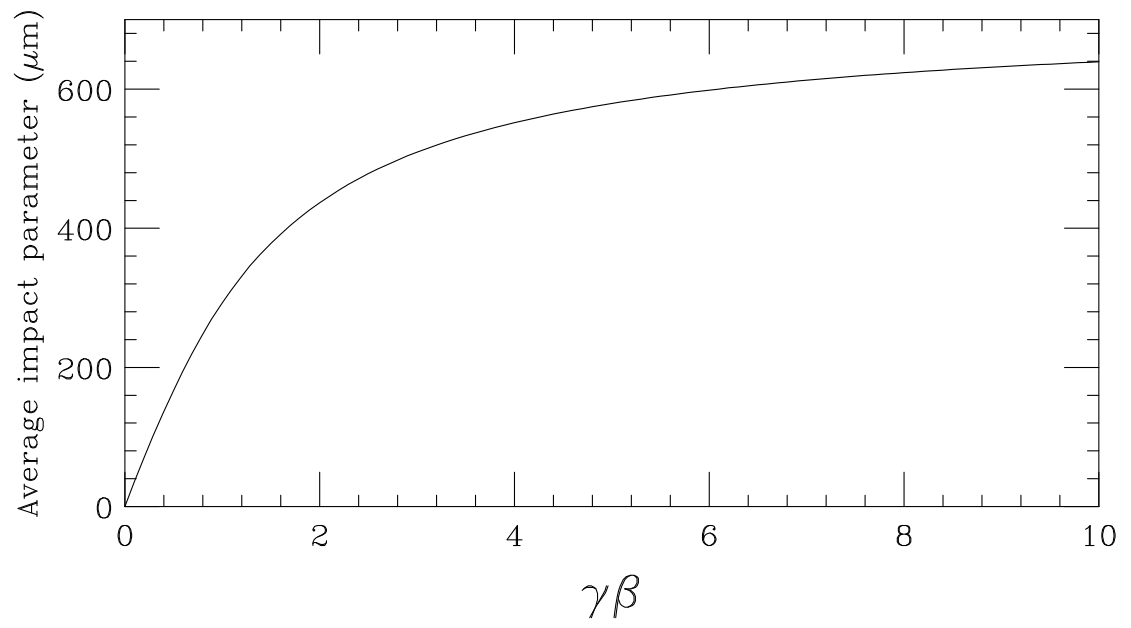

Figure 2: Dependence of $\langle b\rangle$ on $\gamma \beta$ [3].

Table 1: Fraction of events remaining for a given $\langle b\rangle$ when events with $b>b_{0}$ are discarded [3].

\begin{tabular}{lccccc}
\hline \hline $\begin{array}{c}b_{0}(\mu \mathrm{m}) \\
\langle b\rangle(\mu \mathrm{m})\end{array}$ & 100 & 200 & 300 & 400 & 500 \\
\hline 150 & 0.237 & 0.542 & 0.748 & 0.866 & 0.930 \\
300 & 0.080 & 0.237 & 0.400 & 0.542 & 0.658 \\
450 & 0.040 & 0.129 & 0.237 & 0.347 & 0.450 \\
\hline \hline
\end{tabular}

boost are denoted by $\theta^{*}$ in the $B$ rest frame and $\theta$ in the lab. The transformation between them is $\sin \theta=\sin \theta^{*} /\left[\gamma\left(1+\beta \cos \theta^{*}\right)\right]$. The isotropy of muon emission in $\cos \theta^{*}$ can be used to calculate the average values of $\sin \theta$ and $b=\gamma \beta \sin \theta c \tau$, where $c \tau=450 \mu \mathrm{m}$ and

$$
\langle\sin \theta\rangle=\frac{1}{2} \int_{0}^{\pi} \frac{\sin ^{2} \theta^{*} d \theta^{*}}{\gamma\left(1+\beta \cos \theta^{*}\right)}=\frac{\pi}{2} \frac{1}{1+\gamma} .
$$

The dependence of $\langle b\rangle$ on $\gamma \beta$ is shown in Fig. 2.

An eyeball fit to the CDF $b$ distribution [16] gives $\langle b\rangle=350 \mu \mathrm{m}$. Table 1 denotes the effect of discarding events with $b$ exceeding various values of $b_{0}$.

The D0 Collaboration defines a transverse impact parameter $b_{\perp}$ relative to the closest primary vertex and a longitudinal distance $b_{\|}$from the point of closest approach to this vertex. They choose $b_{\perp}<3000 \mu \mathrm{m}$ and $b_{\|}<5000 \mu \mathrm{m}$. These are related to $b$ as follows. The transverse and longitudinal components of muon momentum in the lab are $p_{\perp}^{\mu}=p^{\mu} \sin \psi, p_{\|}^{\mu}=p^{\mu} \cos \psi$. The distance $d$ of a point along the $\mu$ trajectory from the vertex is $d^{2}=b_{\perp}^{2}+(s \sin \psi)^{2}+\left(s \cos \psi-b_{\|}\right)^{2}$, where $s=$ is the distance along the $\mu$ trajectory from the transverse point of closest approach. The minimum of $d$ is $b=d_{\min }=\left[b_{\perp}^{2}+\left(b_{\|} \sin \psi\right)^{2}\right]^{1 / 2}$. Little signal reduction is seen with $b_{\perp}<500 \mu \mathrm{m}$, $b_{\|}<500 \mu \mathrm{m}[2]$, but we advocate a tighter cut. The key question remains with regard to D0 muons: 
are they really from $b$ decays? This question should be answered by imposing an upper bound of $b_{0}<100 \mu \mathrm{m}$ on the impact parameter $b_{0}$.

\section{What do triple products in $B_{(s)} \rightarrow V_{1} V_{2}$ measure?}

A spinless particle decaying to four spinless particles gives rise to three independent momenta in its rest frame. One can form a T-odd expectation value out of (e.g.) $\mathbf{p}_{\mathbf{1}} \times \mathbf{p}_{\mathbf{2}} \cdot \mathbf{p}_{\mathbf{3}}$ [4, 17]. A famous example is the asymmetry of $(13.6 \pm 1.4 \pm 1.5) \%$ in $K_{L} \rightarrow \pi^{+} \pi^{-} e^{+} e^{-}$reported by the $\mathrm{KTeV}$ Collaboration [18]. However, what if two or more of the final-state particles are identical?

Consider the double-Dalitz decay of a CP-mixture (like $K_{L}$ ) to $e^{+} e^{-} e^{+} e^{-}$. (see, e.g., [19]). For low $M\left(e^{+} e^{-}\right)$this process is like $K_{L} \rightarrow \gamma \gamma$, with photons having relative linear polarizations $(\|, \perp)$ for $\mathrm{CP}=(+,-)$. Interference between $\mathrm{CP}$-even and -odd decays can give a non-vanishing value of $\langle\sin \phi \cos \phi\rangle$, where $\phi$ is the angle between normals to the $e^{+} e^{-}$planes.

Now consider the case of $B \rightarrow V_{1} V_{2}$, with each $V$ decaying to two pseudoscalar mesons $P$. (For an extensive discussion of the formalism, see [20].) One extracts triple products (TPs) from angular analyses:

$$
A_{T} \equiv \frac{\Gamma(\mathrm{TP}>0)-\Gamma(\mathrm{TP}<0)}{\Gamma(\mathrm{TP}>0)+\Gamma(\mathrm{TP}<0)} ; \mathrm{TP} \equiv p_{1} \cdot\left(p_{2} \times p_{3}\right)
$$

they are tiny in the SM. A true T-violation is signified by

$$
\mathscr{A}_{T}^{\text {true }} \equiv \frac{\Gamma(\mathrm{TP}>0)+\bar{\Gamma}(\mathrm{TP}>0)-\Gamma(\mathrm{TP}<0)-\bar{\Gamma}(\mathrm{TP}<0)}{\Gamma(\mathrm{TP}>0)+\bar{\Gamma}(\mathrm{TP}>0)+\Gamma(\mathrm{TP}<0)+\bar{\Gamma}(\mathrm{TP}<0)} .
$$

The matrix element for $B(p) \rightarrow V_{1}\left(k_{1}, \varepsilon_{1}\right)+V_{2}\left(k_{2}, \varepsilon_{2}\right)$ can be written

$$
M=a \varepsilon_{1}^{*} \cdot \varepsilon_{2}^{*}+\frac{b}{m_{B}^{2}}\left(p \cdot \varepsilon_{1}\right)\left(p \cdot \varepsilon_{2}\right)+i \frac{c}{m_{B}^{2}} \varepsilon_{\mu v \rho \sigma} p^{\mu} q^{v} \varepsilon^{* \rho} \varepsilon^{* \sigma} ; q \equiv k_{1}-k_{2}
$$

The transversity amplitudes depend on $a, b, c$ as $A_{\|}(a), A_{0}(a, b)$, and $A_{\perp}(c)$. Under CP conjugation, $a \rightarrow \bar{a}, b \rightarrow \bar{b}, i c \rightarrow-i \bar{c}$. Angular distributions depend on the angle $\phi$ and polar angles $\theta_{1}, \theta_{2}$, each in the rest frame of the decaying $V_{1}$ or $V_{2}$ :

$$
\begin{array}{r}
\frac{d \Gamma}{d \cos \theta_{1} d \cos \theta_{2} d \phi} \sim\left|A_{0}\right|^{2} \cos ^{2} \theta_{1} \cos ^{2} \theta_{2}+(1 / 2)\left|A_{\perp}\right|^{2} \sin ^{2} \theta_{1} \sin ^{2} \theta_{2} \sin ^{2} \phi \\
+(1 / 2)\left|A_{\|}\right|^{2} \sin ^{2} \theta_{1} \sin ^{2} \theta_{2} \cos ^{2} \phi+(1 / 2 \sqrt{2}) \operatorname{Re}\left(A_{0} A_{\|}^{*}\right) \sin 2 \theta_{1} \sin 2 \theta_{2} \cos \phi \\
-(1 / 2 \sqrt{2}) \operatorname{Im}\left(A_{\perp} A_{0}^{*}\right) \sin 2 \theta_{1} \sin 2 \theta_{2} \sin \phi-(1 / 2) \operatorname{Im}\left(A_{\perp} A_{\|}^{*}\right) \sin ^{2} \theta_{1} \sin ^{2} \theta_{2} \sin 2 \phi .
\end{array}
$$

The last two terms are T-odd and of two distinct types.

The interfering amplitudes are characterized by a weak phase difference $\phi_{w}$ and a strong phase difference $\delta$. In addition to the "true" TP $\mathscr{A}_{T}^{\text {true }}$ defined above, one can define [4] a "fake" TP:

$$
\mathscr{A}_{T}^{\text {fake }}=\frac{\Gamma(\mathrm{TP}>0)-\bar{\Gamma}(\mathrm{TP}>0)-\Gamma(\mathrm{TP}<0)+\bar{\Gamma}(\mathrm{TP}<0)}{\Gamma(\mathrm{TP}>0)+\bar{\Gamma}(\mathrm{TP}>0)+\Gamma(\mathrm{TP}<0)+\bar{\Gamma}(\mathrm{TP}<0)}
$$


Table 2: Longitudinal and transverse fractions $f_{L}$ and $f_{T}$ for some $b \rightarrow s$-penguin $B \rightarrow V V$ processes.

\begin{tabular}{ccccc}
\hline \hline & $B_{s} \rightarrow \phi \phi$ & $B^{+} \rightarrow \phi K^{*+}$ & $B^{+} \rightarrow \rho^{0} K^{*+}$ & $B^{0} \rightarrow \rho^{0} K^{* 0}$ \\
& {$[22]$} & {$[23]$} & {$[24]$} & {$[24]$} \\
\hline$f_{L}$ & $0.348 \pm 0.041 \pm 0.021$ & $0.49 \pm 0.05 \pm 0.03$ & $0.52 \pm 0.10 \pm 0.04$ & $0.57 \pm 0.09 \pm 0.08$ \\
$f_{T}$ & $0.652 \pm 0.041 \pm 0.021$ & $0.51 \pm 0.05 \pm 0.03$ & $0.48 \pm 0.10 \pm 0.04$ & $0.43 \pm 0.09 \pm 0.08$ \\
\hline \hline
\end{tabular}

where $\mathrm{TP}_{\text {true }} \propto \sin \phi_{w} \cos \delta, \mathrm{TP}_{\text {fake }} \propto \cos \phi_{w} \sin \delta$. The two T-odd observables are

$$
A_{T}^{(1)} \equiv \frac{\operatorname{Im}\left(A_{\perp} A_{0}^{*}\right)}{\left|A_{0}\right|^{2}+\left|A_{\|}\right|^{2}+\left|A_{\perp}\right|^{2}}, A_{T}^{(2)} \equiv \frac{\operatorname{Im}\left(A_{\perp} A_{\|}^{*}\right)}{\left|A_{0}\right|^{2}+\left|A_{\|}\right|^{2}+\left|A_{\perp}\right|^{2}} .
$$

For CP conjugates, one has similar definitions with barred amplitudes and a minus sign from complex conjugation of the imaginary coefficient of $c$. The TP asymmetries $\mathscr{A}_{T}$ then satisfy

$$
\mathscr{A}_{T}^{\text {true }} \propto \operatorname{Im}\left(A_{\perp} A_{i}^{*}-\bar{A}_{\perp} \bar{A}_{i}^{*}\right), \mathscr{A}_{T}^{\text {fake }} \propto \operatorname{Im}\left(A_{\perp} A_{i}^{*}+\bar{A}_{\perp} \bar{A}_{i}^{*}\right), \quad(i=0, \|) .
$$

The observables $A_{T}^{(1,2)}$ are related to those in Dorigo's talk [21] by " $u$ " $\leftrightarrow A_{T}^{(2)}$; " $v " \leftrightarrow A_{T}^{(1)}$; he reports on their measurement in $B_{s} \rightarrow \phi \phi$.

The decays $B \rightarrow \phi K^{*}$ and $B_{s} \rightarrow \phi \phi$ are both dominated by the $b \rightarrow s$ penguin diagram. Factorization predicts dominant longitudinal polarization of the vector mesons, in contrast to observations $[22,23,24]$ (Table 2). By contrast, the tree-dominated decay $B^{0} \rightarrow \rho^{+} \rho^{-}$has $f_{L}=$ $0.992 \pm 0.024_{-0.013}^{+0.026}[25]$, or nearly 1 as predicted. There is no reason to trust factorization for the penguin amplitude, which may be due to rescattering from charm-anticharm intermediate states.

From $B^{0} \rightarrow \phi K^{* 0}$ amplitudes quoted by [4] we estimate

$$
A_{T}^{(1)}=-0.260 \pm 0.048 ; \bar{A}_{T}^{(1)}=0.203 \pm 0.050 ; A_{T}^{(2)}=0.005 \pm 0.070 ; \bar{A}_{T}^{(2)}=0.010 \pm 0.064 .
$$

These values imply a large fake $A_{T}^{(1)}$ ( since $A_{T}^{(1)}-\bar{A}_{T}^{(1)} \neq 0$ ); no true $A_{T}^{(1)}$ (since $A_{T}^{(1)}+\bar{A}_{T}^{(1)}$ is consistent with zero); and no fake or true $A_{T}^{(2)}$ ( since both $A_{T}^{(2)}$ and $\bar{A}_{T}^{(2)}$ are consistent with zero). The large fake $A_{T}^{(1)}$ simply reflects the importance of strong final-state phases.

\section{5. $B_{s} \rightarrow J / \psi \phi$ vs. $B_{s} \rightarrow J / \psi f_{0}$}

Helicity or transversity analysis for $B_{s} \rightarrow J / \psi \phi$ (S-, P-, D-wave) is avoided for $B_{s} \rightarrow J / \psi f_{0}$ (pure $\mathrm{P}$-wave). As $\mathrm{CP}(J / \psi)=\mathrm{CP}\left(f_{0}\right)=+$, the overall final state is $\mathrm{CP}$ odd. An estimate of the rate for this process [26] is

$$
R_{f_{0} / \phi} \equiv \frac{\Gamma\left(B_{s} \rightarrow J / \psi f_{0}, f_{0} \rightarrow \pi^{+} \pi^{-}\right)}{\Gamma\left(B_{s} \rightarrow J / \psi \phi, \phi \rightarrow K^{+} K^{-}\right)} \simeq 20 \%
$$

to be compared with experimental values $0.252_{-0.032-0.033}^{+0.046+0.027}$ [27], $\simeq 0.18(\sim 30 \%$ stat. error $)$ [28], and $0.292 \pm 0.020 \pm 0.017$ [21]. The CKM structure for this process is the same as for $B_{s} \rightarrow J / \psi \phi$. Although $f_{0}$ decays mainly to $\pi \pi$, it seems to be "fed" mainly from $s \bar{s}$ : Comparing $J / \psi \rightarrow \phi \pi \pi$ and $J / \psi \rightarrow \omega \pi \pi$ [29], one sees a $\pi \pi$ peak at $M\left(f_{0}\right) \simeq 980 \mathrm{MeV}$ in $\phi \pi \pi$, not $\omega \pi \pi$. 


\section{New physics constraints}

Two (of $\sim 100$ ) theoretical analyses [30,31] emphasize the correlation between $a_{s l}^{q}, \Delta m_{q}, \Delta \Gamma_{q}$, and the mixing angle $\phi_{q}$, where $A_{s l}^{b}=(0.506 \pm 0.043) a_{s l}^{d}+(0.494 \pm 0.043) a_{s l}^{s}$. The questions of whether $\beta_{s}$ or $a_{s l}^{q}$ are nonstandard are separate; they are related by $a_{s l}^{q}=\left(\left|\Delta \Gamma_{q}\right| / \Delta m_{s}\right) \tan \phi_{q}$. If the D0 dimuon asymmetry is mainly from $a_{s l}^{s}$, Ref. [31] finds $a_{s l}^{s}=(-12.5 \pm 4.8) \times 10^{-3}$ by combining with the D0 measurement $(-1.7 \pm 9.1) \times 10^{-3}$. Using in this formula the (CDF, LHCb) average $\Delta m_{s}=(17.70 \pm 0.08) \mathrm{ps}^{-1}$ and the (CDF, D0) average $\Delta \Gamma_{s}=0.094 \pm 0.031 \mathrm{ps}^{-1}$, one expects $\phi_{s}=\left(-67_{-7}^{+18}\right)^{\circ}$. Comparing with $\phi_{M}^{s}=(-39 \pm 17)^{\circ}$, this would favor slightly larger $\Delta \Gamma_{s}$ or a nonstandard value of $a_{s l}^{d}$. In Ref. [5] it is noted that one must respect the SM prediction of $\Delta m_{q}$. New physics must affect mainly phases of mixing amplitudes.

\section{A cursory look at new physics scenarios}

Supersymmetry has generic flavor-changing (but controllable) effects [32]. Randall-Sundrum [33] scenarios in which different quarks lie at different points along a fifth dimension offer a language for understanding quark mixings; but there is no predictive scheme yet. Theories with an extra (flavor-changing) $Z$ can induce mixing as desired. In Ref. [31] a contribution to $\Delta \Gamma$ is introduced through a new light pseudoscalar (an on-shell state in $B_{s} \leftrightarrow \bar{B}_{s}$ ). These are just some examples of a wealth of models on the market. Some of them predict other observable consequences but there are too many to enumerate exhaustively. Two of my current favorites are (1) a fourth generation, and (2) a hidden sector.

Lunghi and Soni [34] note the tension between $\sin 2 \beta=\sin 2 \phi_{3}=0.668 \pm 0.023$ (measured in $B$ decays) and that $(0.867 \pm 0.048)$ in (their) CKM fit. They note effects of new physics on both $\Delta$ Flavor $=1$ (penguin) and $\Delta$ Flavor $=2$ (box) amplitudes but give no specifics on $\beta_{s}$ or $a_{s l}^{s}$.

In a "hidden sector" let an extended gauge sector $G$ describe dark matter, and let there be particles $Y$ with charges in both the SM and in $G$, and particles $X$ with charges only in $G$. A box diagram describing $B_{s}-\bar{B}_{s}$ mixing in this scenario is shown in Fig. 3. Table 3 gives examples of ordinary, mixed, and "shadow" matter. There are clearly many opportunities in such a scenario for new contributions to penguin and box diagrams.

Table 3: Types of matter and their SM and hidden charges.

\begin{tabular}{cccc}
\hline \hline Type of matter & Std. Model & $\mathrm{G}$ & Example(s) \\
\hline Ordinary & Charged & Uncharged & Quarks, leptons \\
Mixed $(Y)$ & Charged & Charged & Superpartners \\
Shadow $(X)$ & Uncharged & Charged & $E_{8}^{\prime}$ of $\mathrm{E}_{8} \otimes \mathrm{E}_{8}^{\prime}$ \\
\hline \hline
\end{tabular}

\section{Summary}

$B_{s}$ decays and mixing provide potential mirrors of new physics. While the phase $\beta_{s}$ has moved toward its Standard Model value, even the currently measured value of $\beta_{s}$ should be manifested in 


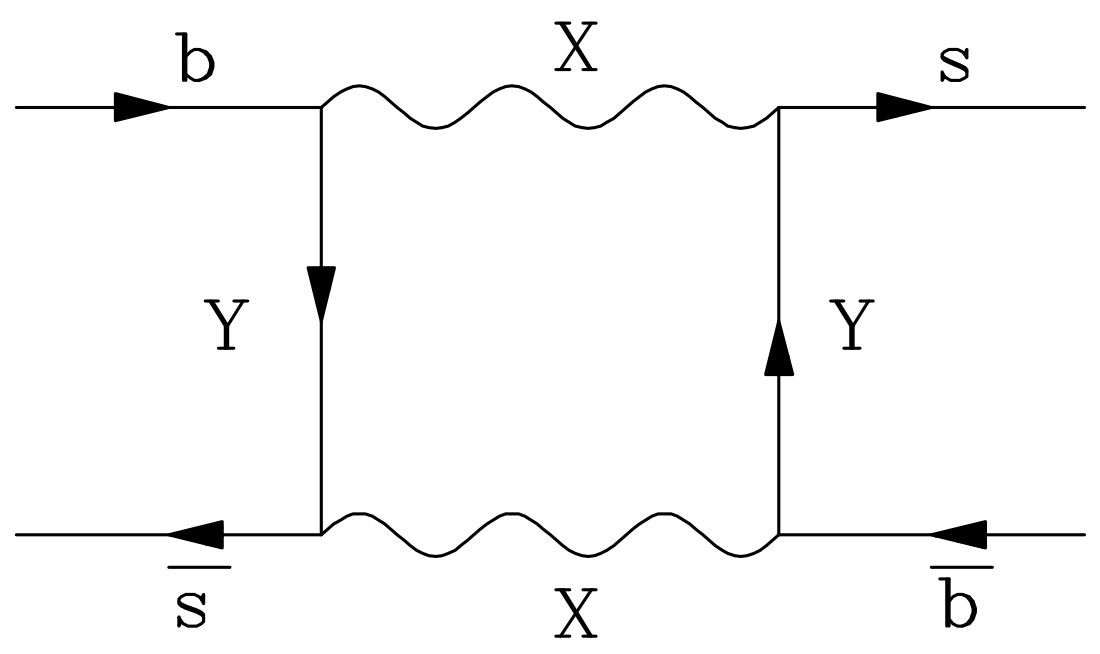

Figure 3: Diagram utilizing a hidden sector describing $B_{s}-\bar{B}_{s}$ mixing.

time-dependent quantities.

The D0 collaboration [2] claims a dimuon charge asymmetry. At this conference [15] CDF has reported a remeasurement of $\bar{\chi}$ and we look forward to their further progress on dimuons. The signal requires subtraction of a big kaon background. Is what's left really due to $b$ quark decays? We have proposed an impact parameter cut of $b<100 \mu \mathrm{m}$ to find out [3].

Using triple products in four-body decays, one can construct T-odd observables providing strong and weak phase information. There is interest in what new physics one can learn from $B_{s} \rightarrow \phi \phi[21]$.

As for whether there is new physics in any of the above hints, I urge you to have your favorite model ready; there are enough to go around.

\section{Acknowledgments}

We are grateful to B. Bhattacharya, M. Dorigo, D. London, and D. Tonelli for helpful discussions. The work of J.L.R. was supported in part by the United States Department of Energy, Grant No. DE-FG02-90ER40560.

\section{References}

[1] M. Gronau and J. L. Rosner, Phys. Lett. B 669, 321 (2008) (arXiv:0808.3761 [hep-ph]).

[2] V. Abazov et al. (D0 Collaboration), Phys. Rev. D 82, 032001 (2010); Phys. Rev. Lett. 105, 081801 (2010).

[3] M. Gronau and J. L. Rosner, Phys. Rev. D 82, 077301 (2010).

[4] A. Datta et al., arXiv:1103.2442v2.

[5] A. Lenz, U. Nierste, and the CKMfitter Collaboration, Phys. Rev. 83, 036004 (2011).

[6] A. Abulencia et al. (CDF Collaboration), Phys. Rev. Lett. 97, 242003 (2006). 
[7] LHCb Collaboration Report No. LHCb-CONF-2011-005, April 7, 2011, presented by Stefania Vecchi at this Conference. See: https://lhcb.web.cern.ch//hcb/temporary/Docs/LHCb-CONF-2011-005.pdf

[8] T. Aaltonen et al. (CDF Collaboration), Phys. Rev. Lett. 100, 161802 (2008).

[9] V. M. Abazov et al. (D0 Collaboration), Phys. Rev. Lett. 101, 241801 (2008).

[10] CDF Public Note CDF/ANAL/BOTTOM/PUBLIC/10206 Version 1.1.

[11] O. Leroy, presented at $2011 \mathrm{La}$ Thuile Meeting on behalf of LHCb, estimates the CDF value as $\phi_{M}=(-31 \pm 29)^{\circ}$.

[12] D0 Collaboration, D0 6098-CONF, quotes a value of $\phi_{M}=\left(-44_{-21}^{+22} \pm 1\right)^{\circ}$.

[13] LHCb Collaboration Report No. LHCb-CONF-2011-006, Feb. 15, 2011, presented by U. Uwer at this Conference.

[14] A. Lenz and U. Nierste, arXiv:1102.4274.

[15] CDF Collaboration, presented by R. Harr, this Conference.

[16] T. Aaltonen et al. (CDF Collaboration), Phys. Rev. D 77, 072004 (2008).

[17] G. Valencia, Phys. Rev. D 39, 3339 (1989).

[18] E. Abouzaid et al. (KTeV Collaboration), Phys. Rev. Lett. 96, 101801 (2006).

[19] G. D. Barr et al. (NA31 Collaboration), Z. Phys. C 65, 361 (1995).

[20] A. Datta and D. London, Int. J. Mod. Phys. A 19, No. 15, 2505 (2004).

[21] CDF Collaboration, presented by M. Dorigo, this Conference, arXiv:1105.4437 [hep-ex]. This value has been updated by the CDF Collaboration subsequent to the Conference.

[22] CDF Collaboration, "Measurement of the Polarization Amplitudes of the $B_{s} \rightarrow \phi \phi$ Decay," CDF Note 10120, March 31, 2011.

[23] B. Aubert et al. (BaBar Collaboration), Phys. Rev. Lett. 99, 201802 (2007).

[24] B. Aubert et al. (BaBar Collaboration), Phys. Rev. Lett. 97, 201801 (2006).

[25] B. Aubert et al. (BaBar Collaboration), Phys. Rev. D 76, 052007 (2007).

[26] S. Stone and L. Zhang, Phys. Rev. D 79, 074024 (2009).

[27] R. Aaij et al. (LHCb Collaboration), Phys. Lett. B 698, 115 (2011).

[28] J. Li et al. (Belle Collaboration), Phys. Rev. Lett. 106, 121802 (2011).

[29] G. J. Feldman and M. L. Perl, Phys. Reports 33C, 285 (1977).

[30] Z. Ligeti et al., Phys. Rev. Lett. 105, 131601 (2010).

[31] Y. Bai and A. Nelson, Phys. Rev. D 82, 114027 (2010).

[32] W. Altmannshofer, A. J. Buras, and P. Paradisi, Phys. Lett. B 688, 202 (2010); W. Altmannshofer and D. M. Straub, JHEP 1009: 078 (2010).

[33] L. Randall and R. Sundrum, Phys. Rev. Lett. 83, 3370 (1999); Phys. Rev. Lett. 83, 4690 (1999).

[34] E. Lunghi and A. Soni, Phys. Lett. B 697, 323 (2011). 